\title{
Criteria for Fire Risk Ranking
}

JOHN M. WATTS, JR.

Fire Safety Institute

Post Office Box 674

Middlebury, Vermont 05753, USA

\begin{abstract}
Fire risk ranking is evolving as a means of evaluating fire safety that is conducive to the assimilation of research results. This paper summarizes research into the extent, nature, and criteria for fire risk ranking methods. Concepts of fire risk ranking are presented and examples of the major methods which have been developed are briefly described. The methods have similar basic components of parameters, values, and relationships. The nature and limitations found in application of these components are discussed. Ten criteria are presented as a guide to the development and evaluation of fire risk ranking methods. These criteria apply to the basic components and to documentation and output of fire risk ranking systems.
\end{abstract}

KEYWORDS: decision analysis, decision making, fire risk, fire safety evaluation, risk analysis, risk ranking, systems analysis.

\section{INTRODUCTION}

Fire risk ranking is a link between fire science and fire safety. As we learn more about the behavior of fire, it is important that this new knowledge be implemented to meet fire safety goals and objectives. One of the obstructions to implementation of new technology is the dearth of structured fire safety decision making. Fire risk ranking is evolving as a means of evaluating fire safety that is conducive to the assimilation of research results.

As part of an ongoing project to analyze the potential use of fire risk ranking systems, a significant number of approaches have been identified and reviewed [1]. It has been found that while there is wide spread development and use of fire risk ranking techniques there is very little information on methodology or evaluation criteria. This paper is a step toward a protocol for development and evaluation of ranking approaches to fire safety. It presents criteria to be considered in the construction of a fire risk ranking model or in assessment of a specific application. 


\section{Fire Risk}

By nature of the circumstances, fire safety decisions often have to be made under conditions where the data are sparse and uncertain. The technical parameters of fire risk are very complex and normally involve a network of interacting components, the interactions generally being non-linear and multidirectional. However, complexity and sparseness of data do not preclude useful and valid approaches. Such circumstances are not unusual in decision making in business or other risk venues. (Exploration of outer space illustrates how success can be achieved when there are little relevant data.)

Fire risk analysis involves a large number of multifarious factors which are difficult to assess in a uniform and consistent way. The analysis of such complex systems is difficult but not impossible as evidenced by activities in areas such as nuclear safety and environmental protection. Detailed risk assessment can be an expensive and labor intensive process and there is considerable scope for improving the presentation of results. Ranking can provide a cost-effective means of risk evaluation which is sufficient in both utility and validity.

\section{Ranking}

Risk ranking is the process of modeling and scoring causal and mitigating parameters to produce a rapid and simple estimate of relative fire risk. The incentive for risk ranking techniques is to provide decision makers with a transparent and defensible way of arriving at decisions.

An essential characteristic of ranking alternatives is the aggregation of entities into a single index. The theory behind using a single index to represent many non-commensurable features is well developed in decision analysis [2]. The sheer mass of input information makes the use of ranking indices attractive.

\section{Fire Risk Ranking}

Fire risk ranking originated with insurance rating schedules in the 19 th century. However, in the last few decades, there has been a move to develop more wide spread analytical procedures.

In general, fire risk ranking assigns values to selected variables based on professional judgement and past experience. The selected variables represent both positive and negative fire safety features and the assigned values are then operated on by some combination of arithmetic functions to arrive at a single value. This single value can be compared to other similar assessments or to a standard.

Fire risk ranking generally has high utility due to the relative ease of application but lacks validity because of the unspecified nature of the selection of variables and their relationships. The relationship of risk ranking techniques to other forms of fire risk analysis is discussed elsewhere $[3,4]$.

\section{EXAMPLES}

Numerous schemes for using a weighted combination of parameters to provide a ranking of fire risk have been tried. These include systemic applications to 
buildings or elasses of occupaney and applications to more specific components of the fire risk.

Risk ranking approaches have been referred to by various designations such as index systems [5], numerical grading [6], point schemes [7], and rating schedules [3].

Three of the most widely used and most well documented fire risk ranking methods will be briefly described as examples of the approaches taken. They are the US Fire Safety Evaluation System, the Swiss Gretener method and the UK Edinburgh scheme.

\section{Fire Safety Evaluation System}

The Fire Safety Evaluation System (FSES) $[6,8]$ is a method for determining equivaleneies to the NFPA Life Safety Code for certain institutions and other occupancies. The technique was developed at the US Center for Fire Research in the late $1970 \mathrm{~s}$. It has since been regularly adapted to new editions of the Life Safety Code.

The FSES treats risk and safety parameters separately. It begins with a determination of relative risk derived from characteristics of a health care occupancy. Five occupancy risk factors are used; patient mobility, patient density, fire zone location, ratio of patients to attendants, and average patient age. Variations of these factors have been assigned relative weights determined from the experienced judgement of a panel of fire safety experts. Risk is then calculated as the product of the assigned values for the five factors.

The system dictates that the calculated risk be offset by safety features. Thirteen safety parameters were selected. These parameters and their respective ranges of values are also products of the same panel of experts. There is no correlation of these fire safety parameters to the previously defined risk factors.

Alternative fire safety strategies are identified as containment, extinguishment, and people movement. The expert panel's opinion was again employed to determine in a binary fashion which fire safety parameters apply to each fire safety strategy. Values of the parameters are then summed for each strategy with an adjustment for the value of automatic sprinklers for people movement safety. The resulting sums are considered to be the available level of each fire safety strategy.

The calculated level for each fire safety strategy is then compared to predetermined minimum levels. For the category of "general safety" the sum of all available safety parameter values is compared to the occupancy risk previously calculated.

\section{Gretener Method}

M. Gretener of the Swiss Fire Prevention Service developed an arithmetical evaluation of fire risk in buildings $[9,10,11]$. His premise was that determining fire risk by statistical methods based on loss experience was no longer efficient. The Gretener method is important because of its acceptance for insurance rating and code enforcement and because of its simple mathematical formulation. 
The Gretener method expresses parameters of ignition and fire spread and parameters of fire protection as empirically derived numerical values. The product of the hazard parameters gives a value for potential hazard, while the product of the fire protection parameters yields a value for protective measures. The ratio of these products is taken as the measure of expected fire severity.

It is immediately appealing that the approach begins with the explicit concept of risk as the expectation of loss, given by the product of hazard probability and hazard severity:

$$
\mathrm{R}=\mathrm{A} \times \mathrm{B}
$$

where:

$\mathrm{R}=$ fire risk,

$A=$ probability that a fire will start, and

severity.

$B=$ fire hazard, degree of danger, or probable

Thus, the Gretener method is based on these two probabilities and combines them in accordance with probability theory.

A further departure from the more popular approaches to fire risk ranking is the calculation of fire hazard as a ratio rather than a sum.

Fire Hazard $=$ Potential Hazard / Protective Measures

$$
B=P /(N \times S \times F)
$$

where:

$$
\begin{aligned}
& B=\text { fire hazard, } \\
& P=\text { potential hazard, } \\
& N=\text { standard fire safety measures, } \\
& S=\text { special fire safety measures, and } \\
& F=\text { fire resistance of the building. }
\end{aligned}
$$

Potential hazard, $P$, is the product of hazard elements whose magnitudes are influenced on the one hand by the building contents, i.e. materials and merchandise present, and on the other hand by the building itself.

As with most other risk ranking approaches, the values for these individual factors are not based solely on statistics, but are empirical quantities resulting from a comparison of analyses of fire risks for which fire protection measures are either common or required by law.

\section{Edinburgh Model}

A matrix approach to fire safety was developed at the University of Edinburgh, $[12,13,14]$ and has been extended at the University of Ulster $[15,16]$. The original objective of this work was to improve the evaluation of fire safety in UK hospitals through a systematic method of appraisal.

As a logical extension of a single fire safety matrix, such as developed in the FSES, consider that there are more than two categories of fire safety parameters. This suggests a hierarchy of lists of things, or decision making levels, that comprise fire safety. Such a hierarchy is presented as follows: 


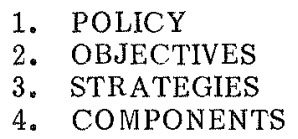

These represent common levels of fire safety decision making but there may be more or fewer in a particular application. For example, an even lower level dealing with individual hardware items could be added, or intermediate levels could be used to better define certain relationships.

This hierarchy of levels of detail of fire safety suggests a series of matrices is appropriate to model the relationships among various fire safety factors, that is, a matrix of policy versus objectives would define a fire safety policy by identifying the specific objectives which are held most desirable. In turn, a matrix of objectives versus strategies would identify the relationship of these factors, and a matrix of strategies versus hardware components would suggest where to use what. Thus, a matrix may be constructed to examine the association of any two adjacent levels in a hierarchy of fire safety factors.

An even more appealing aspect of this approach is that two or more matrices may be combined (multiplied) to produce information on the importance of specific details of building elements to an overall fire safety policy information which has not here to fore been available. This method is the only such grading of fire safety factors with an explicitly defined relationship to fire safety goals and objectives.

Another important contribution of the Edinburgh model is the parameter interaction matrix. Construction of a square matrix of risk parameters provides a systematic approach to the assessment of interdependence of each pair of parameters. This permits adjustment of results to reflect synergisms and other associations of parameters in a consistent manner.

\section{COMPONENTS}

Fire risk ranking methods are found to have three basic components $[1,6]$; a list of parameters, procedures for assigning values to the parameters, and relationships which define mathematical operations on the parameter values to produce an assessment of hazard or risk. Analysis of the nature of these components in the many different approaches has led to identification of common characteristics.

\section{Parameters}

Parameters of fire risk ranking systems, also referred to as factors, variables, etc., identify the ingredients of fire safety. Fire safety in buildings is a complex system with an inordinately large number of factors which may affect it. It is computationally feasible to deal with only a relatively small number of variables. Therefore, it becomes necessary to reduce the large number of variables to an appropriate subset.

It is intuitively appealing to postulate that safety from fire is a Paretian phenomenon in that a relatively small number of factors account for most of the problem. This is supported by general fire loss figures which suggest that a small number of factors are associated with a large proportion of fire deaths. 


\section{Values}

The next component of fire risk ranking is the establishment of quantitative measures associating fire risk with the qualitative characteristics of the parameters. Value selection for the parameters is where the existing methods show the greatest range of variation. Sources for these values range from fire test data to hearsay.

The approach to determination of values can be either objective or subjective. The main criticism of objective estimates is that historical data may not be relevant to the future conditions being considered. For some decisions the subjective approach may be superior when it takes into account both antecedent data and the decision maker's assessment of present and future influences.

There are decision analysis techniques that can be used when, as is most often the case, little or no data are available. Multi-attribute utility theory $[17,18]$ of fers a way, through formal questioning, of developing meaningful values. The analytical hierarchy process $[15,19,20]$ is an even more formal technique that produces mathematical measures of consistency. Delphi $[21,22]$ is less formal and has been used in several fire risk ranking projects including the FSES and the Edinburgh model.

In all but the simplest approaches, two sets of values are associated with each parameter, intensity and importance. Intensity is a measure of the amount or degree that a parameter is present in a specific application, e.g. number of stories, completeness of automatic detection, etc. Importance is a weight indicating the influence or significance of the parameter to fire safety. Most often these are combined into a single dimensionless value.

\section{Relationships}

Relationships are the mechanisms by which the parameter values are combined to yield a measure of fire risk. The general expression is given by equation (1).

$$
R=\operatorname{fCn}\left(a_{i} x_{i}^{b_{i}}\right), \quad i=1,2, \ldots, n
$$

where:

$$
\begin{aligned}
& \mathrm{R}=\text { risk } \\
& \mathrm{x}_{\mathrm{i}}=\text { measure of parameter } \mathrm{i} \\
& \mathrm{a}_{\mathrm{i}}, \mathrm{b}_{\mathrm{i}}=\text { weights of parameter } \mathrm{i} \\
& \mathrm{n}=\text { number of parameters }
\end{aligned}
$$

In most of the fire risk ranking methods studied, the function (fen) used is addition. The risk is measured by the weighted sum of the parameter values as indicated in equation (2) where $b_{i}=1$ for all $i$.

$$
R=\sum_{i=1}^{n}\left(a_{i} x_{i}\right)
$$

This expression implicitly assumes the parameters are all independent in their effect on fire risk. 
The Gretener method uses multiplication as the function by which fire risk is determined from the parameter values. This is expressed in equation (3) where $a_{i}=1$ for all $i$ and $b_{i}=+/-1$ depending on whether the parameter represents a potential hazard or a protective measure.

$$
R=\prod_{i=1}^{n}\left(x_{i}^{b_{i}}\right)
$$

Multiplication of parameter values implicitly assumes that all the parameters are completely interactive. That is, the impact on fire risk of any unit change in a single parameter will be dependent on the values of each of the other parameters.

Most of the fire risk ranking methods studied do not address the interaction of parameters. Yet it is intuitive that certain combinations of parameters, e.g. smoke detection and automatic suppression, are not simply additive in their associated effect on fire risk. At least two methods do deal explicitly with parameter interaction. The FSES uses exceptions in determining parameter values for certain pairs of parameters in combination. The Edinburgh model uses a parameter interaction matrix to systematically assess potential relationships among all the parameters.

Linearity is implicitly assumed by the relationships employed in all the methods studied.

\section{CRITERIA}

The fire protection community seems to be largely unconcerned with the proliferation of fire risk ranking and how it is being used. The available literature deals only with development and application of a specific method or general descriptions of several selected approaches. Like any analytical techniques, risk ranking methods have their limitations and should not be used uncritically.

The purpose of fire risk ranking is to provide a useful aid to decision making. Usefulness requires the methodology to be simple yet credible. It must be easy to apply but sophisticated enough to provide an acceptable minimum of technical validity. Credibility can also be improved through consistency and transparency. The approach should be systematic and clearly discernible to all interested parties that the relevant technical issues have been appropriately covered.

Based on the review of numerous fire risk ranking systems, several criteria are proposed as an aid in the development and evaluation of similar approaches. They are qualitative guidelines that do not guarantee a better result but identify minimal requirements for assessment of the credibility of a fire risk ranking.

Criterion 1: Development and implementation of the method should be thoroughly documented according to standard procedures. One of the hallmarks of professionalism is that as a study proceeds, a record is made of assumptions, data, parameter estimates and why they were chosen, model structure and details, steps in the analysis, relevant constraints, results, sensitivity tests, validation, and so on. Little of this information is available for most fire risk ranking methods. 
In addition to facilitating review, there are other practical reasons not to slight the documentation. 1) If external validation is to be conducted, adequate documentation will be a prerequisite. 2) During the life cycle of a fire risk ranking system the inescapable changes and adjustments will require appropriate documentation. 3) Clear and complete documentation enhances confidence in the method, its absence inevitably carries with it the opposite effect.

The value of the documentation will be improved if it follows established guidelines. Standard formats for documentation are primarily directed at largescale computer models $[23,24]$ but can be readily adapted in principle to more general applications.

Criterion 2: Partition the universe rather than select from it. One of the least well established procedures in fire risk ranking is the choice of parameters. In following a systemic approach e.g. [25] it is best to be comprehensive. In the Edinburgh model, this is achieved by using the NFPA Fire Safety Concepts Tree [26]. The Tree branches out from the holistic concept of fire safety objectives. A cut set on the Tree will then identify a group of parameters which encompasses all possible fire safety features.

Criterion 3: Parameters should represent the most frequent fire scenarios. In determining the level of detail of the parameters, it is necessary to look at those factors which are most significant, statistically or by experienced judgement. This criterion may also be used as an alternative to Criterion 2, providing the need for systemic comprehensiveness is satisfied.

Criterion 4: Provide operational definitions of parameters. If the methodology is to be used by more than a single individual, it is necessary to ensure precise communication of the intent of key terms. Many fire risk parameters are esoteric concepts which have a wide variety of interpretations even within the fire community.

Criterion 5: Elicit subjective values systematically. Most fire risk ranking methods rely heavily on experienced judgement. The use of formalized, documented procedures, such as the previously mentioned multi-attribute utility theory, analytical hierarchy process, and Delphi, significantly increases credibility of the system. Similarly, use of recognizable scaling techniques will enhance credibility.

Criterion 6: Parameter values should be maintainable. One variable that is not explicitly included in fire risk ranking is time. Yet the influence of time is ubiquitous. It influences the fire risk both internally (e.g. deterioration) and externally (e.g. technological developments). In order for a method to have a reasonably useful lifetime, it must be amenable to updating. This implies that the procedure for generating parameter values must be repeatable. Changes over time and new information dictate that the system facilitate revisions.

Criterion 7: Treat parameter interaction consistently. In the majority of cases this will consist of an explicitly stated assumption of no interactive effect among parameters. Where interactions are considered, it is important that they be dealt with systematically to avoid bias. The Edinburgh interaction matrix is one approach to this assessment.

Criterion 8: State the linearity assumption. While this assumption is universal in fire risk ranking, it is also well known that fire risk variables do not necessarily behave in a linear fashion. It is important to the acceptance of ranking methods 
and their limitations that such assumptions are understood.

Criterion 9: Describe fire risk by a single indicator. The objective of most fire risk ranking methods is to sacrifice details and individual features for the sake of making the assessment easier. Information should be reduced to a single score even in the most complex applications. Techniques have been espoused to combine technical, economic, and socio-political factors [27]. The results should be presented in a manner that makes their significance clear in a simple and unambiguous way. Unless all those involved can understand and discuss the meaning of the ranking, there will not be general confidence in its adequacy.

Criterion 10: Validate results. Some attempt should be made to verify that the method does in fact differentiate between lesser and greater fire risks with sufficient precision. The level of accuracy demanded here is not the same as for other engineering purposes, establishing an order of magnitude will generally suffice.

\section{REFERENCES}

1. Watts, John M., Jr., "Fire Risk Rating Schedules", ASTM Symposium on Fire Hazard and Fire Risk Assessment, San Antonio TX, December 1990.

2. Miser, Hugh J., and Edward S. Quade, eds., Handbook of Systems Analysis, North-Holland, New York, 1985.

3. Watts, John M., Jr., "Fire Risk Assessment Schedules", Section 4, Chapter 11, in The SFPE Handbook of Fire Protection Engineering, National Fire Protection Association, Quincy MA, 1988 (pp, 4-89 to 4-102).

4. Hall, John R., Jr., and Ai Sekizawa, "Fire Risk Analysis: General Conceptual Framework for Describing Models", Fire Technology, Vol. 27, No. 1, 1991, pp. $33-53$.

5. Rosenblum, Gary R., and Steven A. Lapp, The Use of Risk Index Systems to Evaluate Risk, in Risk Analysis: Setting National Priorities, proceedings of the Society for Risk Analysis, Houston TX, 1987.

6. Nelson, Harold E., "Overview: Numerical Grading Systems", report from the 1987 workshop on Analytical Methods for Designing Buildings for Fire Safety Design, National Academy Press, Washington DC, 1988.

7. Rasbash, D.J., Approaches to the Measurement and Evaluation of Fire Safety, Third International Fire Protection Engineering Institute, Wageningen, the Netherlands, February 1980.

8. Nelson, H.E., and A.J. Shibe, 1980, "A System for Fire Safety Evaluation of Health Care Facilities," NBSIR 78-1555, Center for Fire Research, National Bureau of Standards, Washington, DC.

9. Gretener, M., "Evaluation of Fire Hazard and Determining Protective Measures", Association of Cantonal Institutions for Fire Insurance (VKF) and Fire Prevention Service for Industry and Trade (BVD), Zurich, 1973.

10. Gretener, M., "Fire Risk Evaluation", Association of Cantonal Institutions for Fire Insurance (VKF), Society of Engineers and Architects (SIA), and Fire 
Prevention Service for Industry and Trade (BVD), Zurich, 1980.

11. Kaiser, J., "Experiences of the Gretener Method", Fire Safety Journal, vol. 2, 1980, pp. 213-222.

12. Marchant, E.W., "Fire Safety Evaluation (Points) Scheme for Patient Areas Within Hospitals", Department of Fire Safety Engineering, University of Edinburgh, 1982.

13. Marchant, E.W., Fire Safety Engineering - A Quantified Analysis, Fire Prevention No. 210, June 1988, pp. 34-38.

14. Stollard, P., "The Development of a Points Scheme to Assess Fire Safety in Hospitals", Fire Safety Journal, Vol. 7, No. 2, 1984, pp. 145-153.

15. Shields, T.J., and G.W. Silcock, An Application of the Analytic Hierarchical Process to Fire Engineering, Fire Safety Journal, Vol. 11, 1986, pp. 235-242.

16. Donegan, H.A., T.J. Shields, and G.W. Silcock, "A Mathematical Strategy to Relate Fire Safety Evaluation and Fire Safety Policy Formation for Buildings", Fire Safety Science - Proceedings of the Second International Symposium, He misphere, 1989, pp. 433-441.

17. Raiffa, Howard, Decision Analysis, Addison-Wesley, Reading MA, 1968.

18. Keeny, R.L., and H. Raiffa, Decisions with Multiple Objectives: Preferences and Value Tradeoffs, Wiley, New York, 1976.

19. Saaty, T.L., The Analytical Hierarchy Process, McGraw-Hill, New York, 1980 .

20. Golden, B.L., E.A. Wasil, and P.T. Harker, eds., The Analytic Hierarchy Process, Springer-Verlag, New York, 1989.

21. Linstone, Harold A., and Murray Turoff, eds., The Delphi Method: Techniques and Applications, Addison-Wesley, Reading MA, 1975.

22. Harmathy, T.Z., "The Delphi Method - A Complement to Research", Fire and Materials, Vol. 6, , No. 2, 1982 , pp. 76-79.

23. Gass, S.I., Documenting a Computer Based Model, Interfaces, Vol. 14, No. 3, 1984, pp. 84-93.

24. ASTM, Standard Guide for Documentation of Computer Software for Fire Models, ASTM, Philadelphia, 1990 (Draft).

25. Beard, Alan N., "Towards a Systemic approach to Fire Safety", Fire Safety Science - Proceedings of the First International Symposium, Hemisphere, 1986, pp. 943-952.

26. NFPA 550, Guide to the Fire Safety Concepts Tree, National Fire Protection Association, Quincy MA, 1986.

27. Chicken, John C., and Michael R. Hayns, The Risk Ranking Technique in Decision Making, Pergamon Press, Oxford, 1989. 